\title{
Self-Reconfigurable Molecule Robots as 3D Metamorphic Robots
}

\author{
Craig McGray Daniela Rus \\ Department of Computer Science \\ Dartmouth College \\ Hanover, NH 03755
}

\begin{abstract}
This paper describes a three-dimensional selfreconfiguring system that is capable of reconfiguration and the associated planning in polynomial time. The approach is to reduce a system composed of Molecule Robots [KRVM] to the Metamorphic Robots of [PaChSCh]. Having done so, we are able to apply polynomial-time planning algorithms that have previously been used only in two-dimensional systems.
\end{abstract}

\section{Introduction}

A self-reconfiguring robotic system consists of a set of modules that can autonomously change their relative positions in order to change the shape of the overall system. Self-reconfiguring systems can offer greater versatility and greater fault tolerance than conventional fixed-architecture systems.

Because of the distributed nature and large number of components of a self-reconfiguring robot, the geometric planning required to reconfigure from one shape to another can be complex. In [ChPa], Chirikjian, et. al. present a polynomial-time solution to the planning problem for a particular class of selfreconfiguring systems known as Metamorphic Robots.

One quality of a Metamorphic Robot that simplifies the planning process is that the centers of all of its modules conform to a regular lattice. Additionally, any module on a continuous surface of the robot can move to any lattice point on that same continuous surface, without requiring any other modules to move.

[MKuKo] and [PaChSCh] describe two-dimensional systems that possess the above two qualities. While the algorithms of [ChPa] generalize to the threedimensional case, mechanical constraints make it more difficult to produce a module with the above qualities that is capable moving in three-dimensions

In [KRVM], we describe a three-dimensional selfreconfiguring system. However, the modules in this system, called Molecule Robots, do not have the two qualities of Metamorphic Robots that are mentioned above. So, the general planning problem for this system remains to be solved.

In this paper we construct a three-dimensional meta-module by composing 16 Molecule Robots. The resulting meta-modules connect to one another in such a way that their centers conform to a cubic lattice. We show how this module can make transitions that allow it to traverse any continuous surface of a configuration. This allows us to use the algorithms of [ChPa] to plan reconfigurations for structures composed of these three-dimensional modules.

\section{Previous Work}

Related work in designing modular robots includes [PaKh95, PaKh93, FuKa, ChBu, Yim, NeSa, HaSa]. These papers focus on how to design and build robotic systems out of modules from a small set of component types, and how to reconfigure these systems by changing the ways in which the components are used.

self-reconfiguring robots are a special kind of modular robot. These robots are capable of reconfiguring without external intervention. Previous work in self-reconfiguring robotics includes [MKuKo, YMTKuKo, PaChSCh, ChPa, KRVM]. [MKuKo, YMTKuKo, $\mathrm{PaChSCh}, \mathrm{ChPa}$ ] consider twodimensional self-reconfiguring systems. These systems have properties that allow the application of known planning algorithms for self-reconfiguring systems. [KRVM] presents a three-dimensional selfreconfiguring system. General planning algorithms for this system are not known.

This paper builds on the above references to present a three-dimensional system with properties that allow the use of polynomial-time motion planning algorithms.

\section{The Molecule Robot}

A Molecule Robot [KRVM], shown in Figure 1 consists of two cubic components called atoms linked by a rigid connection called a bond. Each atom has five 
inter-Molecule connection points and two degrees of freedom. One degree of freedom allows the atom to rotate relative to its bond connection, and the other degree of freedom allows the atom (thus the entire Molecule) to rotate relative to exactly one of the interMolecule connectors. This latter rotational degree of freedom cannot be about the connector opposite the bond connection.

A Molecule Robot can make twelve different elementary transitions. Due to symmetry, these twelve transitions may be divided into the three types shown in Figures 2.

Transitions that would disconnect the configuration are disallowed, as are transitions that would result in collisions. Actuators must be positioned as described in [KRVM] before a transition can occur.

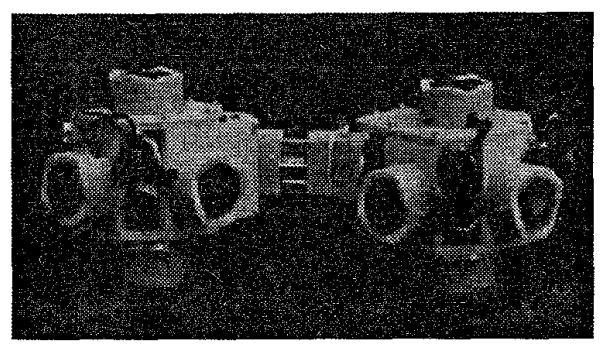

Figure 1: The Molecule Robot is composed of two atoms, connected by a right-angle rigid bond. The robot has 4 degrees of freedom: two rotational degrees of freedom about the bond and one rotational degree of freedom on each atom about a single inter-Molecule connector.

\section{Definitions}

In Section 5 we construct a meta-module consisting of 16 Molecule Robots, and describe a reduction from this meta-module to a Metamorphic Robot system. This allows us to use the planning algorithms described in [ChPa] to reconfigure systems composed of these meta-modules. The following definitions are needed to show the meta-module construction.

\subsection{Module Connections}

Two modules, $x$ and $y$, are connected if either of the following conditions holds:

- $x$ is attached to $y$.

- $x$ is attached to some module, $z$, and $z$ is connected to $y$.

A set, $S$, of modules is said to be connected if every module in $S$ is connected to every other module in $S$.

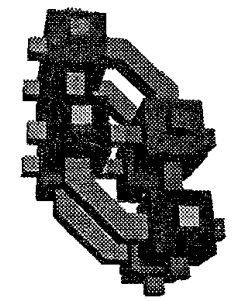

starting position

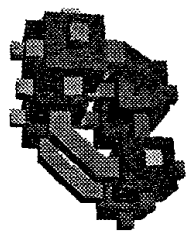

$\mathrm{x}$-axis rotation

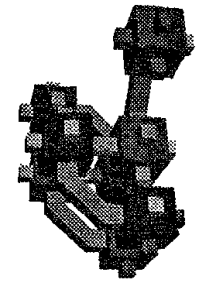

$\mathrm{y}$-axis rotation

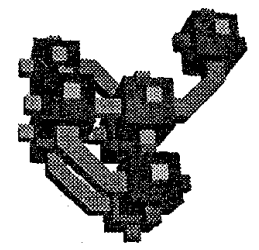

z-axis rotation
Figure 2: The Molecule Robot's three basic transitions. One atom of the moving robot remains stationary, while the other atom and the bond move around it. The above three transitions are geometrically equivalent to rotating the robot $90^{\circ}$ relative to the stationary atom's $\mathrm{x}$-axis, $\mathrm{y}$-axis, and $\mathrm{z}$-axis, respectively.

\subsection{Module Positions}

A module position is a placement and orientation in the space of discourse. Two positions are said to be adjacent if modules placed in those positions could attach. Two positions are said to intersect if modules placed in those positions would have at least one point in common in the space of discourse.

\subsection{Module Transitions}

A transition is a change in a module's position, expressed as the difference between the module's previous position and its new position. Each transition is associated with a certain set of preconditions that must be true before the transition can be performed.

\subsection{Module Classes}

A module class is defined as a set of module transitions with their preconditions.

\subsection{Configurations}

A configuration, $C=\{M, B, P\}$ is a set of modules, $M$, associated via a bijection, $B$, to a set of connected, non-intersecting module positions. Positions are expressed not in absolute terms, but rather as offsets from an arbitrary module in $M$. 
Two configurations, $C_{1}=\left\{M_{1}, B_{1}, P_{1}\right\}$ and $C_{2}=$ $\left\{M_{2}, B_{2}, P_{2}\right\}$ are said to be equivalent iff there is a bijection $f: M_{1} \leftrightarrow M_{2}$ such that $\forall m \in M, B_{1}(m)=$ $B_{2}(f(m))$.

\subsection{Reconfiguration}

Let $K$ be a class of module, with transition set $T$. A configuration, $C_{1}$, of $n$ modules of class $K$, is said to be reconfigurable to another configuration, $C 2=$ $\left\{M_{2}, B_{2}, P_{2}\right\}$ of $n$ modules of class $K$, if one of the following conditions holds:

- $C_{1}$ is equivalent to $C_{2}$.

- There is a transition, $t \in T$ and a module, $m \in M_{1}$ such that applying $t$ to $m$ yields a configuration, $C_{3}$, that is reconfigurable to $C_{2}$.

\section{A Reduction For Motion Planning}

In this section, we show how to construct a robot that is capable of polynomial-time selfreconfiguration. We do so by composing 16 Molecule Robots into into a meta-module called the Axes module. We then show that Axes modules satisfy the conditions required to use polynomial-time planning algorithms described in [ChPa].

The rest of this section proceeds as follows. We describe a hierarchy of three modules. Each of the latter two modules is composed of multiple copies of the module at the previous level in the hierarchy. We show that each module can "move" by separating into its underlying components, moving the underlying components, and then re-assembling in a new position. We use this to define a set of feasible transitions for the modules. With each level in the hierarchy, the new transitions add flexibility to the self-reconfiguring system.

The first module we consider is the Molecule Robot. We show how Molecule Robots can be aggregated to construct a new module called a Double-Pair. We then show how Double-Pair modules can be aggregated to construct an Axes module. Finally, we show that systems composed of Axes modules satisfy the requirements of the polynomial-time planning algorithms described in [ChPa].

\subsection{The Double-Pair Module}

Lemma 1 (composite-lemma) Let $K_{1}$ be a class of modules that have cubic geometry and that can connect to identical modules across each of six faces. Let a module, $m$, of class $K_{1}$ be capable of diagonal and straight transitions within a cubic lattice, provided that the following preconditions hold:

- Diagonal Transition

1. The removal of $m$ will not disconnect the configuration.
2. There is no module in the destination cell, $d$, to which $m$ is moving.

3. There is a module, $h$, adjacent to $m$, that is also adjacent to $d$.

4. There is an empty cell, e, adjacent to $m$, that is also adjacent to $d$.

5. Let $P$ be the plane defined by $\{m, d, h\}$. There must be a plane, $P I$ adjacent to $P$, such that the four cells in $P I$ that are adjacent to $\{m, d, h, e\}$ are empty.

- Straight Transition:

1. The removal of $m$ will not disconnect the configuration.

2. There is no module in the destination cell, $d$, to which $m$ is moving.

3. There is a module, $h_{1}$, adjacent to $m$.

4. There is a module, $h_{2}$, adjacent to $h_{1}$, that is also adjacent to d.

5. Let $P$ be the plane defines by $m, d, h_{1}$. There must be a plane, PI adjacent to $P$, such that the two cells in $P /$ that are adjacent to $m, d$ are empty.

Four Molecule Robots can be aggregated to implement a module of class $K_{1}$.

Let a Double-Pair module be the configuration of 4 Molecule Robots, as shown in Figure 3. As discussed in [KRVM], each atom and each bond of a Molecule Robot in any configuration occupies exactly one cell of a cubic lattice, whose cell size is equal to the bounding box of an atom. So, a Double-Pair module occupies a bounding box that is exactly twelve times the volume of a Molecule Robot's atom. 20 of the Double-Pair module's inter-Molecule connection points lie on the surface of this bounding box.

We therefore define a lattice where each cell will exactly fit a Double-Pair module. Imagine that this lattice is colored red and black like a $3 \mathrm{D}$ checker board. If Double-Pair modules in black cubes are always "right-side-up", and modules in red cubes are always "up-side-down" (see Figure 3), then any two adjacent Double-Pair modules can connect across at least two inter-Molecule connection points. So, DoublePair modules have cubic geometry, and can connect to other Double-Pair modules across all six faces.

Now, we need to show that Double-Pair modules can execute the $K_{1}$ transitions described above.

There are 6 straight directions in which a module of class $K_{1}$ can move. Given a direction of movement, there are 4 directions in which the helper modules $\left(h_{1}\right.$ and $h_{2}$ ) could lie. Given a direction of movement 


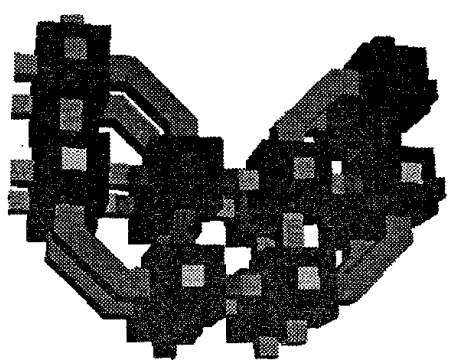

Figure 3: Two adjacent Double-Pair modules. The module on the left is "right-side-up", while the module on the right is "up-side-down". The Double-Pair module is composed of 4 Molecule Robots, and is a member of class $K_{1}$.

and a direction for the helpers, there are two directions in which the empty adjacent plane $(P t)$ could lie. So, there are a total of 48 straight reconfigurations that must be demonstrated. Due to symmetries in the Double-Pair module, only 12 of these reconfigurations are distinct.

Similarly, there are 12 diagonal directions in which a module of class $K_{1}$ can move. Given a direction of movement, there are 2 directions in which the helper module $(h)$ could lie, and 2 directions in which $P$, could lie. So, there are 48 diagonal reconfigurations that must be demonstrated. Due to symmetries in the Double-Pair module, only 6 of these are distinct.

We have discovered the sequences of Molecule transitions that are required in order to show that the above 18 distinct reconfigurations are possible. We have verified the correctness of these sequences through Prolog simulation. One of these sequences is abbreviated in Figure 4. For a more complete discussion, please see our technical report [McRu].

\subsection{The Axes Module}

Lemma 2 (composite-lemma) Let $K_{2}$ be a class of modules, each of which occupies one cell in a cubic lattice, and can connect to identical modules across each of six faces. Let any module, $m$, be capable of diagonal and straight transitions, provided that the following preconditions hold:

\section{- Straight Transition:}

1. The removal of $m$ will not disconnect the configuration.
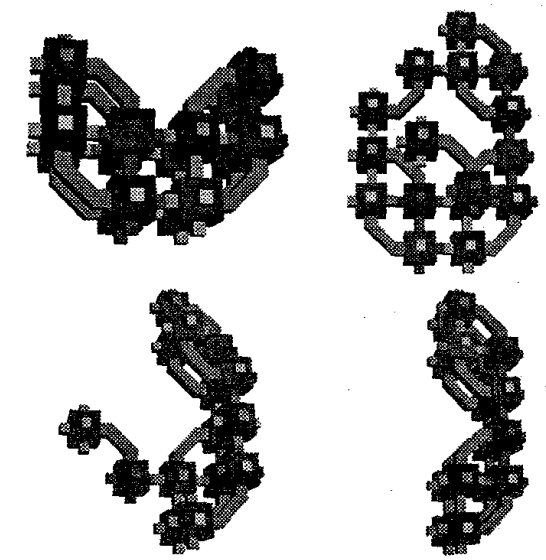

Figure 4: A $K_{1}$ diagonal transition in the $x-y$ plane, implemented with Molecule Robots as component modules. The transition requires 64 Molecule transitions and is not necessarily optimal.

2. The destination cell, $d$, is empty.

3. There is a module, $h_{1}$, adjacent to $m$.

4. There is a module, $h_{2}$, adjacent to $d$, that is also adjacent to $h_{1}$.

\section{- Diagonal Transition}

1. The removal of $m$ will not disconnect the configuration.

2. The destination cell, $d$, is empty.

3. There is a module, $h$, adjacent to $m$, that is also adjacent to $d$.

4. There is an empty cell, $e$, adjacent to $m$, that is also adjacent to d.

Four Modules of class $K_{1}$ can be aggregated to form a module of class $\mathrm{K}_{2}$.

Let an Axes module be a configuration of four modules of class $K_{1}$, as shown in Figure 5 . Since modules of class $K_{1}$ conform to a rectangular lattice, $L$, with cell size $\left(L_{x}, L_{y}, L_{z}\right)$, the bounding box of an Axes module is $\left(2 L_{x}, 2 L_{y}, 2 L_{z}\right)$. So, Axes modules conform to a lattice, $L /$ with cell size $\left(2 L_{x}, 2 L_{y}, 2 L_{z}\right)$.

Given an Axes module with 4 components of class $K_{1}$, call the component that is adjacent to three others the Origin. Call the remaining components the $\mathrm{X}$-axis, $\mathrm{Y}$-axis, and $\mathrm{Z}$-axis, respectively. Let $m_{1}$ and $m_{2}$ be two Axes modules in adjacent cells of $L I$. The Origin of one module must be adjacent to either the $\mathrm{X}$-axis, the $\mathrm{Y}$-axis, or the Z-axis of the other module. So, since any module of class $K_{1}$ can attach to any adjacent 


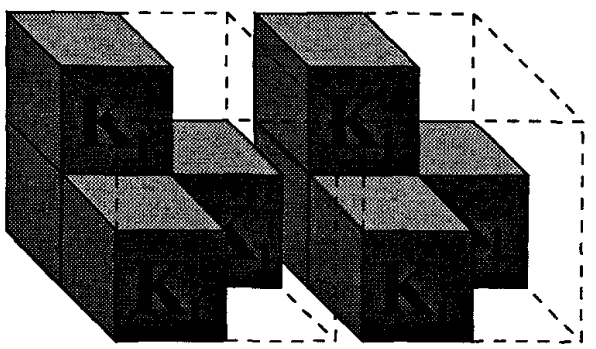

Figure 5: Two adjacent Axes modules. Each Axes module is composed of four modules of class $K_{1}$, and is a member of class $K_{2}$. In the above Figure, the $\mathrm{x}$-axis of the left module can connect to the origin of the module on the right. Any two Axes modules in adjacent cells can connect in this fashion.

\section{K3 Straight Transition:}

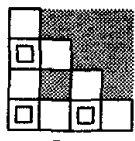

Start

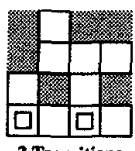

2 Transitions

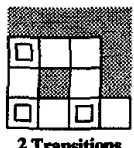

2 Transitions

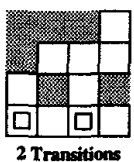

2 Transitions
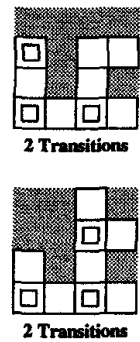

Figure 6: A straight transition of a module of class $K_{2}$, implemented with component modules of class $K_{1}$. Each frame depicts two layers of $K_{1}$ modules: small squares represent modules in the top level, and large squares represent modules in the bottom level.

module of class $K_{1}$, any two adjacent modules of class $K_{2}$ must also be able to attach.

Now, we need to show that Axes modules can reconfigure in such a way as to execute the $K_{2}$ transitions described above. There are 24 straight reconfigurations and 24 diagonal reconfigurations that must be demonstrated. However, all of the straight reconfigurations are symmetric, and all of the diagonal transitions are symmetric. So, only two distinct sequences of $K_{1}$ transitions are required.

We have discovered two such sequences (one straight, and one diagonal), and have documented them in our technical report, $[\mathrm{McRu}]$. The straight transition is simple, and is abbreviated in Figure 6 .

Since Axes modules occupy a 3-dimensional rectan- gular lattice, can attach to any adjacent Axes module, and can make straight and diagonal transitions provided the preconditions of $K_{2}$ are met, we know that we are able to implement a module of class $K_{2}$ with 4 modules of class $K_{1}$.

Lemma 3 (composite-theorem) Modules of class $K_{2}$ can self-reconfigure in polynomial time.

Proof: We can separate the cells in a lattice that are not in a configuration into connected components. In [ChPa], Chirikjian, et. al. argue that if any movable module adjacent to the exterior of a configuration can always move to any cell in the exterior of the configuration that is in the same connected component as its starting cell, without moving any other modules, then reconfiguration of a system composed of such modules can be performed in $O\left(n^{2}\right)$ transitions. Polynomialtime algorithms to achieve this bound are provided in [ChPa].

Since modules of class $K_{2}$ can make straight transitions alongside other modules, and can make diagonal transitions around other modules, they can traverse the connected exterior of a configuration as discussed in $[\mathrm{ChPa}$.

So, motion planning for self-reconfiguring systems of modules of class $K_{2}$ can be performed in polynomial time.

Theorem 4 There exists a three-dimensional class of module whose transitions can be described as a composition of Molecule transitions, and that is selfreconfiguring in polynomial time.

Proof: Given lemmas 1 and 2, we know that 4 Molecule Robots can aggregate to a module of class $K_{1}$, and that 4 modules of class $K_{1}$ can aggregate to a module of class $K_{2}$. So, we can transitively construct a module of class $K_{2}$ from 16 Molecule Robots. Since lemma 3 showed that systems of modules of class $K_{2}$ can reconfigure in polynomial time, Molecule Robots can be aggregated to form a module that defines a polynomial-time self-reconfiguring system.

\section{Discussion}

We have shown that Molecule Robots are sufficient as building blocks to construct polynomial-time selfreconfiguring robotic systems in three dimensions.

We have demonstrated how to construct such a system using 16 Molecule Robots per module. We know from [ChPa] that the number of Molecule transitions required to reconfigure such a system is $O\left(n^{2}\right)$. 


\section{Future Work}

Three primary advantages of the Molecule Robot for constructing self-reconfiguring robotic systems, are that the robot module is small, simple, and inexpensive. In order to achieve polynomial-time planning for our system, we have aggregated 16 Molecule Robots into each module in our system. This results in a trade-off between the size/simplicity/cost of our system, and the ability to use tractable planning algorithms. We are interested in minimizing this trade-off.

So, two interesting questions are: What is the minimum number of Molecule Robots required to satisfy the restrictions of known planning algorithms? Do there exist polynomial-time planning algorithms that do not require these restrictions?

While the $O\left(n^{2}\right)$ bound for reconfiguration is asymptotically tight with respect to the minimum number of transitions required, it is not necessarily tight with respect to the amount of time required. Could we use parallel transitions to improve on the $O\left(n^{2}\right)$ time bound? What is the greatest attainable speedup?

\section{Acknowledgements}

We are grateful to Keith Kotay and Marsette Vona for insightful discussions. We are grateful to Brian Locke for his help and advice regarding the rapid prototyping machine which we used to build the Molecule Robot.

This paper describes research done in the Dartmouth Robotics Laboratory. Support for this work was provided through the NSF CAREER award IRI9624286 and the NSF award IRI-9714332.

\section{References}

[FuKa] T. Fukuda and Y. Kawauchi, Cellular robotic system (CEBOT) as one of the realization of self-organizing intelligent universal manipulator, in Proceedings of the 1990 IEEE Conference on Robotics and Automation, pp. 662-667.

[HaSa] G. Hamlin and A. Sanderson, Tetrabot modular robotics: prototype and experiments, in Proceedings of the IEEE/RSJ International Symposium of Robotics Research, pp 390-395, Osaka, Japan, 1996.

[KRVM] K. Kotay, D. Rus, M. Vona, C. McGray The Self-reconfiguring Robotic Molecule.

[McRu] C. McGray, D. Rus Dartmouth College Technical Report Number PCS TR98-342
[MKuKo] S. Murata, H. Kurokawa, and Shigeru Kokaji, Self-assembling machine, in Proceedings of the 1994 IEEE International Conference on Robotics and Automation, San Diego, 1994.

[NeSa] B. Neville and A. Sanderson, Tetrabot family tree: modular synthesis of kinematic structures for parallel robotics, in Proceedings of the IEEE/RSJ International Symposium of Robotics Research, pp 382-390, Osaka, Japan, 1996.

[PaChSCh] A. Pamecha, C-J. Chiang, D. Stein, and G. Chirikjian, Design and implementation of metamorphic robots, in Proceedings of the 1996 ASME Design Engineering Technical Conference and Computers in Engineering Conference, Irvine, CA 1996.

[PaKh95] C. Paredis and P. Khosla, Design of Modular Fault Tolerant Manipulators, in The First Workshop on the Algorithmic Foundations of Robotics, eds. K. Goldberg, D. Halperin, J.-C. Latombe, and R. Wilson, pp 371-383, 1995.

[PaKh93] C. Paredis and P. Khosla, Kinematic Design of Serial Link Manipulators from Task Specifications, in International Journal of Robotic Research, Vol. 12, No. 3, pp 274-287; 1993.

[Yim] M. Yim, A reconfigurable modular robot with multiple modes of locomotion, in Proceedings of the 1993 JSME Conference on Advanced Mechatronics, Tokyo, Japan 1993.

[YMTKuKo] E. Yoshida, S. Murata, K. Tomita, H. Kurokawa, and S. Kokaji, Distributed Formation Control of a Modular Mechanical System, in Proceedings of the 1997 International Conference on Intelligent Robots and Systems, Grenoble, France, 1997.

[ChBu] I. Chen and J. Burdick, Enumerating the Non-Isomorphic Assembly Configurations of a Modular Robotic System, to appear in the International Journal of Robotics Research.

[CLBD] R. Cohen, M. Lipton, M. Dai, and B. Benhabib, Conceptual design of a modular robot, Journal of Mechanical Design, March 1992, pp. 117-125.

[ChPa] G. Chirikjian, Amit Pamecha Bounds for Metamorphic Robots. 